\title{
UNIFORM CONVERGENCE OF DENSITY ESTIMATORS ON SPHERES
}

Non-parametric estimation of a probability density for random variables taking values on an $s$-dimensional unit sphere is studied in [1], [5], [6]. The object of the present paper is to establish new uniform convergence theorems for several estimators: we use successively the histogram method, the spherical cap and the kernel methods. In part D, we present simulation results.

Let $\mathcal{D}$ be the set of continuous densities, defined on the sphere $S$; we estimate $f$, an element of $\mathcal{D}$, from a sample of size $n$, denoted by $X_{1}, \ldots, X_{n}$. The density $f$ satisfies $\int_{S} f(x) d \mu(x)=1$, where $\mu$ is the Lebesgue measure on $S$.

A. The histogram estimator. We are going to describe a partition of the sphere which will allow us to use the main theorem of [4].

This theorem establishes a necessary and sufficient condition for uniform convergence - in probability and almost completely - using the histogram estimator on a metric space, for every $f$ in $\mathcal{D}$. To use it for $S$, it will be sufficient to construct a sequence $\Delta_{k(n)}$ of partitions $\Delta_{k}=\left\{\Delta_{k, r}: r \in R_{k}\right\}$, the Borel sets $\Delta_{k, r}$ being such that

$$
\begin{gathered}
\lim _{k \rightarrow \infty} \sup _{r \in R_{k}}\left(\operatorname{diam} \Delta_{k, r}\right)=0, \quad \lim _{k \rightarrow \infty} \sup _{r \in R_{k}}\left(\operatorname{area} \Delta_{k, r}\right)=0, \\
\limsup _{k \rightarrow \infty} \frac{\sup _{r \in R_{k}}\left(\operatorname{area} \Delta_{k, r}\right)}{\inf _{r \in R_{k}}\left(\operatorname{area} \Delta_{k, r}\right)}<\infty .
\end{gathered}
$$

We choose the integer $k(n)$ such that $\lim _{n \rightarrow \infty} k(n)=+\infty$. For $r \in R_{k}$, let $\nu_{n, r}$ be the number of $X_{i}$ 's belonging to $\Delta_{k, r}$.

1991 Mathematics Subject Classification: 62G07, 62G20.

Key words and phrases:histogram estimator, spherical cap estimator, kernel estimator. 
The histogram estimator $\widehat{f}_{n}$ is given by

$$
\forall r \in R_{k}, \forall x \in \Delta_{k, r}, \quad \widehat{f}_{n}(x)=\frac{\nu_{n r}}{n \mu\left(\Delta_{k, r}\right)},
$$

$\mu\left(\Delta_{k, r}\right)$ denoting the area of $\Delta_{k, r}$. With these notations, the main theorem of [4] states that $\widehat{f}_{n}$ is uniformly convergent, in probability and almost completely, if and only if

$$
\left[\inf _{r \in R_{k}} \mu\left(\Delta_{k, r}\right)\right]^{-1}=o(n / \log n) \quad \text { where } k=k(n) .
$$

First, we are going to construct the partition for $s=3$. Then we shall explain it for any $s$.

1. Partition for $s=3$. A parametric representation of $S$ is

$$
\begin{array}{ll}
x_{1}=\cos \theta_{1}, & \theta_{1} \in[0, \pi], \\
x_{2}=\sin \theta_{1} \cos \theta_{2}, & \\
x_{3}=\sin \theta_{1} \sin \theta_{2}, & \theta_{2} \in[0,2 \pi[.
\end{array}
$$

The "poles" of $S$, corresponding to $\theta_{1}=0$ and $\theta_{1}=\pi$, must belong to a unique element of the partition, so we define the Borel sets $\Delta_{k, r}=\Delta_{k, r_{1}, r_{2}}$ in the following manner:

$$
\begin{aligned}
\Delta_{k, 0}= & {\left[0, \arccos \left(1-1 / k^{2}\right)[\times[0,2 \pi[,\right.} \\
\Delta_{k, 1, r_{2}}= & {\left[\arccos \left(1-1 / k^{2}\right), \arccos (1-2 / k)\left[\times\left[\left(r_{2}-1\right) \pi / k, r_{2} \pi / k[\right.\right.\right.} \\
& \text { for } r_{2}=1, \ldots, 2 k, \\
\Delta_{k, r_{1}, r_{2}}= & {\left[\arccos \left(1-2\left(r_{1}-1\right) / k\right), \arccos \left(1-2 r_{1} / k\right)\left[\times\left[\left(r_{2}-1\right) \pi / k, r_{2} \pi / k[\right.\right.\right.} \\
\text { for } r_{1}=2, \ldots, k-1 ; r_{2}=1, \ldots, 2 k, & \\
\Delta_{k, k, r_{2}}= & {\left[\arccos (-1+2 / k), \arccos \left(-1+1 / k^{2}\right)\left[\times\left[\left(r_{2}-1\right) \pi / k, r_{2} \pi / k[\right.\right.\right.} \\
\Delta_{k, k+1}= & \text { for } r_{2}=1, \ldots, 2 k,
\end{aligned}
$$

these intervals being closed when necessary. Then we can easily see that, for each $\Delta_{k, r}, \mu\left(\Delta_{k, r}\right)$ is equivalent to $2 \pi / k^{2}$, and that there are $2 k^{2}+2$ elements in the partition. The necessary and sufficient condition is then

$$
k^{2}=o(n / \log n) .
$$

2. Construction for arbitrary s. A parametric representation of $S$ is: for $\theta_{i} \in[0, \pi]$ when $i=1, \ldots, s-2$ and $\theta_{s-1} \in[0,2 \pi[$,

$$
\begin{aligned}
x_{1} & =\cos \theta_{1}, \\
x_{i} & =\prod_{j=1}^{i-1} \sin \theta_{j} \cos \theta_{i}, \quad i=2, \ldots, s-1,
\end{aligned}
$$




$$
x_{s}=\prod_{j=1}^{s-1} \sin \theta_{j}
$$

In $\mathbb{R}^{s}$, the distance between two points $M$ and $M^{\prime}$ belonging to the sphere, associated with $\left(\theta_{i}\right)_{i=1, \ldots, s-1}$ and $\left(\theta_{i}^{\prime}\right)_{i=1, \ldots, s-1}$, is

$$
d^{2}\left(M, M^{\prime}\right)=4 \sum_{i=1}^{s-1} \prod_{j=1}^{i-1} \sin \theta_{j} \sin \theta_{j}^{\prime} \sin ^{2} \frac{\theta_{i}-\theta_{i}^{\prime}}{2} .
$$

We notice that, for $i=1, \ldots, s-2, \sin \theta_{i}=0$ implies that $\theta_{i+1}, \ldots, \theta_{s-1}$ are arbitrary.

The area of a part $S^{\prime} \subset S$ is

$$
\mu\left(S^{\prime}\right)=\int_{S^{\prime}} \prod_{i=1}^{s-1} \sin ^{m_{i}} \theta_{i} d \theta_{i} \quad \text { with } m_{i}=s-1-i ; i=1, \ldots, s-1 .
$$

For positive integers $q \geq 0$, define

$$
I_{q}=\int_{0}^{\pi / 2} \sin ^{2 q+1} \theta d \theta, \quad J_{q}=\int_{0}^{\pi} \sin ^{2 q} \theta d \theta .
$$

First, let us construct the elements which do not contain the poles - i.e. the points such that, for one index $i=1, \ldots, s-2, \sin \theta_{i}=0$. These elements can be written as

$$
\Delta_{k, r}=\prod_{i=1}^{s-1}\left[\alpha_{r_{i}-1}, \alpha_{r_{i}}\left[, \quad r \in R_{k}^{\prime}\right.\right.
$$

We choose the values $\alpha_{r_{i}}, i=1, \ldots, s-1$, in the following manner. Consider the integral

$$
\int_{\alpha_{r_{i}-1}}^{\alpha_{r_{i}}} \sin ^{m_{i}} \theta_{i} d \theta_{i}
$$

If $m_{i}=2 q_{i}+1$ with $q_{i} \in \mathbb{N}$, then define

$$
F_{q_{i}}(\alpha)=\int_{0}^{\alpha} \sin ^{2 q_{i}+1} \theta_{i} d \theta_{i} \quad \text { for } \alpha \in[0, \pi] .
$$

Then $F_{q_{i}}(\alpha)$ is increasing from 0 to $F_{q_{i}}(\pi)=2 I_{q_{i}}$; we define $\alpha_{r_{i}}$ from

$$
F_{q_{i}}\left(\alpha_{r_{i}}\right)=\frac{2 r_{i}}{k} I_{q_{i}} \quad \text { for } r_{i}=1, \ldots, k .
$$

Then

$$
\int_{\alpha_{r_{i}-1}}^{\alpha_{r_{i}}} \sin ^{2 q_{i}+1} \theta_{i} d \theta_{i}=\frac{2}{k} I_{q_{i}}
$$


If $m_{i}=2 q_{i}$ with $q_{i} \in \mathbb{N}^{*}$, then define

$$
G_{q_{i}}(\alpha)=\int_{0}^{\alpha} \sin ^{2 q_{i}} \theta_{i} d \theta_{i} \quad \text { for } \alpha \in[0, \pi] .
$$

Then $G_{q_{i}}(\alpha)$ is increasing from 0 to $J_{q_{i}}$; we define $\alpha_{r_{i}}$ from

$$
G_{q_{i}}\left(\alpha_{r_{i}}\right)=\frac{r_{i}}{k} J_{q_{i}} \quad \text { for } r_{i}=1, \ldots, k .
$$

Then

$$
\int_{\alpha_{r_{i}-1}}^{\alpha_{r_{i}}} \sin ^{2 q_{i}} \theta_{i} d \theta_{i}=\frac{1}{k} J_{q_{i}} .
$$

For $m_{i}=0$, i.e. $i=s-1$, we choose

$$
\left[\alpha_{r_{s-1}-1}, \alpha_{r_{s-1}}\left[=\left[\left(r_{s-1}-1\right) \pi / k, r_{s-1} \pi / k\left[, \quad r_{s-1}=1, \ldots, 2 k .\right.\right.\right.\right.
$$

Using the values of $I_{q_{i}}$ and $J_{q_{i}}$, we can easily see that for $r_{i}=2, \ldots, k-1$; $i=1, \ldots, s-2 ;$ and $r_{s-1}=1, \ldots, 2 k$,

$$
\mu\left(\Delta_{k, r}\right)=\frac{C(s)}{k^{s-1}},
$$

where $C(s)$ is a constant; its value follows from the preceding formulations. The whole partition is constructed by generalization of the method explained for $s=3$. When, for an index $i=1, \ldots s-2, \sin \theta_{i}=0$, the associated element of the partition satisfies: $\theta_{i+1}, \ldots, \theta_{s-2}$ are in $[0, \pi]$, and $\theta_{s-1}$ in $[0,2 \pi[$; the intervals for $\theta_{1}, \ldots, \theta_{i}$ are chosen to make the area of $\Delta_{k, r}$ equivalent to the preceding expression.

EXAMPLE (for $s=4$ ). For $r_{1}=2, \ldots, k-1 ; r_{2}=2, \ldots, k-1$; and $r_{3}=1, \ldots, 2 k$,

$$
\begin{aligned}
& \Delta_{k, r}= {\left[\alpha_{r_{1}-1}, \alpha_{r_{1}}[\right.} \\
& \times\left[\arccos \left(1-2\left(r_{2}-1\right) / k\right), \arccos \left(1-2 r_{2} / k\right)\left[\times\left[\left(r_{3}-1\right) \pi / k, r_{3} \pi / k[,\right.\right.\right. \\
& \mu\left(\Delta_{k, r}\right)=\pi^{2} / k^{3},
\end{aligned}
$$

$\alpha_{r_{1}}$ being given from

$$
\frac{1}{2} \alpha_{r_{1}}-\frac{1}{4} \sin ^{2} \alpha_{r_{1}}=\frac{r_{1}}{2 k} \pi
$$

and

$$
\begin{aligned}
\Delta_{k, 0} & =\left[0,(3 \pi / 4)^{1 / 3} / k[\times[0, \pi] \times[0,2 \pi[,\right. \\
\Delta_{k, k+1} & =\left[\pi-(3 \pi / 4)^{1 / 3} / k, \pi\right] \times[0, \pi] \times[0,2 \pi[, \\
\Delta_{k, 1,0} & =\left[(3 \pi / 4)^{1 / 3} / k, \alpha_{1}[\times[0, \sqrt{2} / k[\times[0,2 \pi[,\right. \\
\Delta_{k, 1, k+1} & =\left[(3 \pi / 4)^{1 / 3} / k, \alpha_{1}[\times[\pi-\sqrt{2} / k, \pi] \times[0,2 \pi[,\right.
\end{aligned}
$$




$$
\begin{aligned}
\Delta_{k, 1,1, r_{3}}= & {\left[(3 \pi / 4)^{1 / 3} / k, \alpha_{1}[\times[\sqrt{2} / k, \arccos (1-1 / k)[\right.} \\
& \times\left[\left(r_{3}-1\right) \pi / k, r_{3} \pi / k\left[, \quad r_{3}=1, \ldots, 2 k,\right.\right.
\end{aligned}
$$

and so on.

The number of elements in the partition is

$$
K_{n, 4}=2+k\left(2 k^{2}+2\right)=2 k^{3}+2 k+2 .
$$

Coming back to the general case, we have

$$
K_{n, s}=2 k^{s-1}+2 \frac{k^{s-2}-1}{k-1} .
$$

The necessary and sufficient condition is then

$$
k^{s-1}=o(n / \log n) .
$$

B. The spherical cap estimator. For the sphere $S$ in $\mathbb{R}^{s}$, the spherical cap estimator is defined as in [6].

With each $x \in S$, we associate the spherical cap with pole $x$ and radius $h_{n}$, denoted by $B_{n, x}$; here $h_{n}$ is a sequence of positive real numbers such that

$$
\lim _{n \rightarrow \infty} h_{n}=0
$$

The area of $B_{n, x}$ is

$$
\mu\left(B_{n, x}\right)=C_{s} h_{n}^{s-1}+o\left(h_{n}^{s-1}\right), \quad \text { where } C_{s}=\frac{2 \pi^{(s-1) / 2}}{(s-1) \Gamma((s-1) / 2)} .
$$

We estimate the density $f$ in the following manner. Let $\nu_{n, x}$ be the number of $X_{i}$ 's belonging to $B_{n, x}$. Define

$$
\forall x \in S, \quad \widetilde{f}_{n}(x)=\frac{\nu_{n x}}{n C_{s} h_{n}^{s-1}} .
$$

We are going to prove the following theorem:

For each element $f \in \mathcal{D}, \widetilde{f}_{n}$ is uniformly convergent-in probability and almost completely - if and only if

$$
h_{n}^{1-s}=o(n / \log n) .
$$

Proof of the "if" part. We suppose that

$$
h_{n}^{1-s}=o(n / \log n),
$$

and we are going to prove that, for every $f$ in $\mathcal{D}, \widetilde{f}_{n}$ converges almost completely to $f$, uniformly on $S$.

Let $x$ be an element of $S$, and $\widetilde{f}_{n}(x)$ the associated estimator. We choose $0 x_{1}=0 x$. Let $k_{n}=\left[1 / h_{n}\right]$. Then

$$
\frac{k_{n}}{k_{n}+1}<k_{n} h_{n} \leq 1 \text {. }
$$


Now, $k_{n}$ being chosen, we construct the partition as in part A; $x$ belongs to $\Delta_{k_{n}, 0}$, and the corresponding histogram estimator is

$$
\widehat{f}_{n, k_{n}}(x)=\frac{\nu_{n, 0}\left(k_{n}\right)}{n \mu\left(\Delta_{k_{n}, 0}\right)}, \quad \text { where } \nu_{n, 0}\left(k_{n}\right) \text { is the number of } X_{i} \text { 's in } \Delta_{k_{n}, 0} \text {. }
$$

We do the same construction with the integer $k_{n}+1$ :

$$
\widehat{f}_{n, k_{n}+1}(x)=\frac{\nu_{n, 0}\left(k_{n}+1\right)}{n \mu\left(\Delta_{k_{n}+1,0}\right)} .
$$

Since $\Delta_{k_{n}, 0}$ (resp. $\Delta_{k_{n}+1,0}$ ) is (by part A) the spherical cap of pole $x$ and radius $1 / k_{n}$ (resp. $1 /\left(k_{n}+1\right)$ ), we can write

or

$$
\frac{\nu_{n, 0}\left(k_{n}+1\right)}{n \mu\left(\Delta_{k_{n}, 0}\right)} \leq \widetilde{f}_{n}(x) \leq \frac{\nu_{n, 0}\left(k_{n}\right)}{n \mu\left(\Delta_{k_{n}+1,0}\right)},
$$

$$
\frac{\mu\left(\Delta_{k_{n}+1,0}\right)}{\mu\left(\Delta_{k_{n}, 0}\right)} \widehat{f}_{n, k_{n}+1}(x) \leq \widetilde{f}_{n}(x) \leq \frac{\mu\left(\Delta_{k_{n}, 0}\right)}{\mu\left(\Delta_{k_{n}+1,0}\right)} \widehat{f}_{n, k_{n}}(x) .
$$

From the choices of $h_{n}$ and $k_{n}$, we claim that $\widehat{f}_{n, k_{n}}$ and $\widehat{f}_{n, k_{n}+1}$ converge to $f$ uniformly almost completely.

Choosing a positive $\eta$, we suppose that the events $\left\{d\left(\widehat{f}_{n, k_{n}}, f\right)<\eta\right\}$ and $\left\{d\left(\widehat{f}_{n, k_{n}+1, f}\right)<\eta\right\}$ are realized. For large $n$

$-\eta+\left[\frac{\mu\left(\Delta_{k_{n}+1,0}\right)}{\mu\left(\Delta_{k_{n}, 0}\right)}-1\right] f(x) \leq \widetilde{f}_{n}(x)-f(x) \leq\left[\frac{\mu\left(\Delta_{k_{n}, 0}\right)}{\mu\left(\Delta_{k_{n}+1,0}\right)}-1\right] f(x)+2 \eta$.

Let $H$ be such that $f<H$. Then, for large $n$,

$$
\left|\frac{\mu\left(\Delta_{k_{n}+1,0}\right)}{\mu\left(\Delta_{k_{n}, 0}\right)}-1\right| H<\eta \text {. }
$$

Thus, for large $n$,

$$
P\left[d\left(\widetilde{f}_{n}, f\right)>3 \eta\right] \leq P\left[d\left(\widehat{f}_{n, k_{n}}, f\right)>\eta\right]+P\left[d\left(\widehat{f}_{n, k_{n}+1}, f\right)>\eta\right] .
$$

The choices of $h_{n}$ and $k_{n}$ imply the convergence of the series on the righthand side.

The uniform and almost complete convergence of $\widetilde{f}_{n}$ to $f$ follows immediately.

Proof of the "only if" part. We suppose that, for every $f$ in $\mathcal{D}$, $\widetilde{f}_{n}$ converges to $f$ uniformly in probability. First, we show $h_{n}^{1-s}=o(n)$.

We choose a coordinate system and we consider the spherical cap with radius $1 / 4$ and pole $x\left(\theta_{1}=0\right)$; we choose $f$ to be an element of $\mathcal{D}$ such that, on this cap, $f$ is an arbitrary positive number $\alpha$.

From this choice of $f$, and from the hypothesis, we get

$$
\lim _{n \rightarrow \infty} P\left[\nu_{n x}=0\right]=0,
$$


that is,

$$
\lim _{n \rightarrow \infty}\left(1-\alpha C_{s} h_{n}^{s-1}\right)^{n}=0,
$$

so that $\lim _{n \rightarrow \infty} n \log \left(1-\alpha C_{s} h_{n}^{s-1}\right)=-\infty$ and thus $h_{n}^{1-s}=o(n)$.

Now, we show that $h_{n}^{1-s}=o(n / \log n)$. Let $\beta$ be fixed in $] 0, \pi / 2[$, and let $S^{\prime}$ be the part of $S$ defined by $\beta \leq \theta_{i} \leq \pi-\beta$ for $i=1, \ldots, s-2$, and $0 \leq \theta_{s-1}<2 \pi$.

Let $k_{n}$ be an integer to be defined later; we construct the corresponding partition (as in part A), and let $\left\{\Delta_{k_{n}, r}: r \in R_{k_{n}}^{\prime}\right\}$ be the set of its elements included in $S^{\prime}$.

For each $\Delta_{k_{n}, r}$, we define its center $\bar{x}_{k_{n}, r}$ as follows. For large $n, \Delta_{k_{n}, r}$ can be written as $\prod_{i=1}^{s-1}\left[\alpha_{r_{i}-1}, \alpha_{r_{i}}\left[\right.\right.$ for every $r \in R_{k_{n}}^{\prime}$. Then $\bar{x}_{k_{n}, r}=\alpha_{r_{i}-1 / 2}$, $i=1, \ldots, s-1$, with

$$
\int_{0}^{\alpha_{r_{i}-1 / 2}} \sin ^{m_{i}} \theta_{i} d \theta_{i}=\left\{\begin{array}{l}
\frac{2 r_{i}-1}{k} I_{q_{i}} \\
\text { or } \\
\frac{2 r_{i}-1}{2 k} J_{q_{i}}
\end{array} \quad \text { for } i=1, \ldots, s-2,\right.
$$

and

$$
\alpha_{r_{s-1}-1 / 2}=\frac{2 r_{s-1}-1}{2 k} \pi .
$$

Consider the distance (in $\mathbb{R}^{s}$ ) from $\bar{x}_{k_{n}, r}$ to the boundary of $\Delta_{k_{n}, r}$. Using the expression for $d\left(M, M^{\prime}\right)$ (part A), we can easily see that there exists a positive constant $C(s, \beta)$ such that

$$
\inf _{r \in R_{k_{n}}^{\prime}} d\left(\bar{x}_{k_{n}, r}, \text { boundary of } \Delta_{k_{n}, r}\right) \geq C(s, \beta)^{1 / 2} / k_{n} .
$$

This implies that, for each $r$ in $R_{k_{n}}^{\prime}, \Delta_{k_{n}, r}$ contains the spherical cap with pole $\bar{x}_{k_{n}, r}$ and radius $C(s, \beta)^{1 / 2} / k_{n}$. Choose $k_{n}=\left[C(s, \beta)^{1 / 2} / h_{n}\right]$. Then, for each $r$ in $R_{k_{n}}^{\prime}, \Delta_{k_{n}, r}$ contains the spherical cap with pole $\bar{x}_{k_{n}, r}$ and radius $h_{n}$, i.e. $B_{n, \bar{x}_{k_{n}, r}}$.

Moreover, by definition of $S^{\prime}, R_{k_{n}}^{\prime}$ has $\left[C^{\prime}(s, \beta) k_{n}^{s-1}\right]$ elements, where $C^{\prime}(s, \beta)$ is a positive number depending only on $s$ and $\beta$.

We choose $f$ in $\mathcal{D}$ with $f=\alpha$ on $S^{\prime}, \alpha$ being an arbitrarily small positive number. From the hypothesis, $\widetilde{f}_{n}$ converges to $f$ uniformly in probability, so

$$
\lim _{n \rightarrow \infty} P\left[d\left(\tilde{f}_{n}, f\right)>\alpha / 2\right]=0 .
$$

If one of the $\Delta_{k_{n}, r}$ included in $S^{\prime}$ contains no $X_{i}$, then neither does the cap $B_{n, \bar{x}_{k_{n}, r}}$ and $\widetilde{f}_{n}\left(\bar{x}_{k_{n, r}}\right)=0$, so $d\left(\widetilde{f}_{n}, f\right) \geq \alpha$. The convergence hypothesis implies

$$
\lim _{n \rightarrow \infty} P\left[\bigcup_{r \in R_{k_{n}}^{\prime}}\left\{\nu_{n, r}\left(k_{n}\right)=0\right\}\right]=0
$$


$\nu_{n, r}\left(k_{n}\right)$ being the number of $X_{i}$ 's belonging to $\Delta_{k_{n}, r}$. That is,

$$
\lim _{n \rightarrow \infty} P\left[\bigcap_{r \in R_{k_{n}}^{\prime}}\left\{\nu_{n, r}\left(k_{n}\right) \geq 1\right\}\right]=1 .
$$

Here, we remind that two events $A$ and $B$ of positive probability are in negative correlation if

$$
P(A \mid B) \leq P(A), \quad \text { that is, } \quad P(A \cap B) \leq P(A) P(B) .
$$

More generally, the events $A_{1}, \ldots, A_{n}$ of positive probability are in negative correlation if

$$
\forall I \subset\{1, \ldots, n\}, \quad P\left[\bigcap_{i \in I} A_{i}\right] \leq \prod_{i \in I} P\left(A_{i}\right),
$$

that is, the realization of one of the $A_{i}$ diminishes the probability that the others are realized.

The events in the intersection several lines above are in negative correlation, thus

$$
\lim _{n \rightarrow \infty} \prod_{r \in R_{k_{n}}^{\prime}} P\left[\nu_{n, r}\left(k_{n}\right) \geq 1\right]=1 .
$$

Then, remembering that $f=\alpha$ on $S^{\prime}$, we have

$$
\lim _{n \rightarrow \infty} \prod_{r \in R_{k_{n}}^{\prime}}\left[1-\left(1-\alpha \mu\left(\Delta_{k_{n}, r}\right)\right)^{n}\right]=1 .
$$

From part A, $\mu\left(\Delta_{k_{n}, r}\right)=C(s) / k_{n}^{s-1}$; taking the logarithm, we obtain, for large $n$,

$$
\forall \alpha>0, \quad 1-\frac{n \alpha C(s)}{k_{n}^{s-2} \log \left[C^{\prime}(s, \beta) k_{n}^{s-1}\right]}<0,
$$

thus

$$
\lim _{n \rightarrow \infty} \frac{k_{n}^{s-1} \log \left[C^{\prime}(s, \beta) k_{n}^{s-1}\right]}{n}=0 .
$$

Using the definition of $k_{n}$ from $h_{n}$, and $h_{n}^{1-s}=o(n)$, we obtain the desired result.

C. The kernel estimator. Let $K$ be a positive function, defined on $\mathbb{R}^{+}$, such that

$$
\int_{0}^{\infty} K(u) u^{(s-3) / 2} d u<\infty
$$

For this function $K$ and for a sequence of positive numbers $h_{n}$ with $\lim _{n \rightarrow \infty} h_{n}=0$ the kernel estimator of $f$ is

$$
\widetilde{f}_{n}(x)=\frac{1}{n h_{n}^{s-1} C_{K, s}\left(h_{n}\right)} \sum_{i=1}^{n} K\left(\frac{1-\left\langle x, X_{i}\right\rangle}{h_{n}^{2}}\right),
$$


where $\left\langle x, X_{i}\right\rangle$ is the scalar product and

$$
C_{K, s}\left(h_{n}\right)=h_{n}^{1-s} \int_{S} K\left(\frac{1-\langle x, y\rangle}{h_{n}^{2}}\right) d \mu(y)
$$

$d \mu(y)$ being the area element on $S$.

The constant $C_{K, s}\left(h_{n}\right)$ does not depend on $x$ and can be written as

$$
C_{K, s}\left(h_{n}\right)=\frac{2 \pi^{(s-1) / 2}}{\Gamma((s-1) / 2)} \int_{0}^{2 / h_{n}^{2}}\left(2 u-u^{2} h_{n}^{2}\right)^{(s-3) / 2} K(u) d u
$$

with

$$
\lim _{n \rightarrow \infty} C_{K, s}\left(h_{n}\right)=\frac{2 \pi^{(s-1) / 2}}{\Gamma((s-1) / 2)} \int_{0}^{\infty}(2 u)^{(s-3) / 2} K(u) d u .
$$

Notice first that if we choose

$$
K(u)=\mathbf{1}_{[0,1 / 2]}(u),
$$

then

$$
C_{K, s}\left(h_{n}\right)=\frac{2 \pi^{(s-1) / 2}}{\Gamma((s-1) / 2)} \int_{0}^{1 / 2}\left(2 u-u^{2} h_{n}^{2}\right)^{(s-3) / 2} d u
$$

that is,

$$
C_{K, s}\left(h_{n}\right)=h_{n}^{1-s} \frac{2 \pi^{(s-1) / 2}}{\Gamma((s-1) / 2)} \int_{0}^{2 \arcsin h_{n} / 2} \sin ^{s-2} \theta d \theta .
$$

From part B, we see that $h_{n}^{s-1} C_{K, s}\left(h_{n}\right)$ is the area of the cap $B_{n, x}$, and thus the estimator $\widetilde{f}_{n}$ defined from that function $K$ is the spherical cap estimator.

We are going to prove two uniform convergence theorems for the kernel estimator: a necessary condition for convergence in probability, and a sufficient condition for almost complete convergence. In the proofs, we will follow the method used in [3]. Thus, we do not give all the details; we just indicate how these methods can be adapted for $S$.

1. Necessary condition for convergence. The theorem is:

Suppose that

$$
\lim _{y \rightarrow \infty} y \int_{y}^{\infty} K(u)(2 u)^{(s-3) / 2} d u=0 .
$$

Then, for every $f$ in $\mathcal{D}$, if $\widetilde{f}_{n}$ converges to $f$ uniformly in probability, then $h_{n}^{1-s}=o(n / \log n)$.

First, we show that

$$
h_{n}^{1-s}=o(n)
$$


As in [3], we suppose that this condition is not satisfied, and we show that, for an element $f$ in $\mathcal{D}, \widetilde{f}_{n}$ does not converge in probability.

If $h_{n}^{1-s}$ is not $o(n)$, there exists a positive $\alpha$ and an infinite subset $N_{1}$ of $\mathbb{N}$ such that

$$
\forall n \in N_{1}, \quad h_{n}^{1-s}>\alpha n .
$$

We define a parametric representation of $S$, and we choose $f$ in $\mathcal{D}$ equal to $\alpha$ on $C$ defined by

$$
C=\left\{x \in S: 0 \leq \theta_{1} \leq \pi / 4 ; \theta_{i} \in[0, \pi], i=1, \ldots, s-2 ; \theta_{s-1} \in[0,2 \pi]\right\} .
$$

Let $H$ be an upper bound of $f$.

We choose a positive number $M$ such that

$$
\int_{M}^{\infty} K(u)(2 u)^{(s-3) / 2} d u<\inf \left(\frac{1}{4}, \frac{\alpha}{4 H}\right) \int_{0}^{\infty} K(u)(2 u)^{(s-3) / 2} d u .
$$

Let

$$
\varrho_{n}=h_{n} \sqrt{2 M}
$$

and let $Q_{n}$ be the cap with pole $\xi\left(\theta_{1}=0\right)$ and radius $\varrho_{n}$. Let $H_{n}$ be the event: no one of the $X_{i}$ 's belongs to $Q_{n}$.

We get

$$
P\left(H_{n}\right)=\left[1-\alpha \mu\left(Q_{n}\right)\right]^{n} .
$$

We use the hypothesis on $h_{n}$ and the choice of $\varrho_{n}$ to obtain

$$
P\left(H_{n}\right)>e^{-2(2 M)^{(s-1) / 2} C_{s}}>0 \quad \text { for large } n \text { in } N_{1} \text {. }
$$

Let $f^{H_{n}}$ be the density of $X$ conditioned by $H_{n}$ :

$$
f^{H_{n}}(x)= \begin{cases}0 & \text { on } Q_{n}, \\ \frac{f(x)}{1-\alpha C_{s}(2 M)^{(s-1) / 2} h_{n}^{s-1}} & \text { on } S-Q_{n} .\end{cases}
$$

Then we bound the mean of $\widetilde{f}_{n}(\xi)$ conditioned by $H_{n}$; as in [3], we obtain

$$
\begin{aligned}
& E\left[\widetilde{f}_{n}(\xi) \mid H_{n}\right] \\
& \quad \leq \frac{\left[2 \pi^{(s-1) / 2} / \Gamma((s-1) / 2)\right] H}{\left(1-\alpha C_{s} \varrho_{n}^{s-1}\right) C_{K, s}\left(h_{n}\right)} \int_{\varrho_{n}^{2} /\left(2 h_{n}^{2}\right)}^{2 / h_{n}^{2}} K(u)\left(2 u-u^{2} h_{n}^{2}\right)^{(s-3) / 2} d u .
\end{aligned}
$$

For large $n$, using $\varrho_{n}^{2} /\left(2 h_{n}^{2}\right)=M$, we get

$$
E\left[\widetilde{f}_{n}(\xi) \mid H_{n}\right] \leq \frac{\left[2 \pi^{(s-1) / 2} / \Gamma((s-1) / 2)\right] H}{\left(1-\alpha C_{s} \varrho_{n}^{s-1}\right) C_{K, s}\left(h_{n}\right)} \int_{M}^{\infty} K(u)(2 u)^{(s-3) / 2} d u,
$$

and, from the definition of $M$, 


$$
\begin{aligned}
& E\left[\widetilde{f}_{n}(\xi) \mid H_{n}\right] \\
& \quad \leq \frac{\alpha}{4\left(1-\alpha C_{s} \varrho_{n}^{s-1}\right)} \frac{\left.2 \pi^{(s-1) / 2} / \Gamma((s-1) / 2)\right] \int_{0}^{\infty} K(u)(2 u)^{(s-3) / 2} d u}{C_{K, s}\left(h_{n}\right)} .
\end{aligned}
$$

Remembering that $\lim _{n \rightarrow \infty} \varrho_{n}=0$ and

$$
\lim _{n \rightarrow \infty} C_{K, s}\left(h_{n}\right)=\frac{2 \pi^{(s-1) / 2}}{\Gamma((s-1) / 2)} \int_{0}^{\infty} K(u)(2 u)^{(s-3) / 2} d u
$$

we obtain, for large $n, E\left[\widetilde{f}_{n}(\xi) \mid H_{n}\right] \leq \frac{1}{4} \alpha(1+\varepsilon)$.

The proof is then as in [3], using the Markov inequality, and the fact that, for large $n$ in $N_{1}, P\left(H_{n}\right)$ is strictly positive.

Now we show

$$
h_{n}^{1-s}=o(n / \log n) .
$$

We suppose that $h_{n}^{1-s}=o(n)$, but that the condition $h_{n}^{1-s}=o(n / \log n)$ is not satisfied. Then there exists a positive $\beta$ and an infinite subset $N_{1}$ of $\mathbb{N}$ such that

$$
\forall n \in N_{1}, \quad h_{n}^{1-s}>\beta n / \log n .
$$

Let $\alpha$ be a positive number, to be made precise further, and let us choose $f$ :

$$
f(x)= \begin{cases}f\left(\theta_{1}, \ldots, \theta_{s-1}\right)=\alpha & \text { on } C=[0, \pi / 2] \times[0, \pi]^{s-3} \times[0,2 \pi[, \\ a \sin \theta_{1}+b & \text { on }[\pi / 2,2 \pi / 3] \times[0, \pi]^{s-3} \times[0,2 \pi[, \\ H & \text { elsewhere }\end{cases}
$$

The constants $a, b, H$ are well known from $\alpha$, using the continuity condition, and $\int_{S} f d \mu=1$. More precisely, we get

$$
H=\frac{d_{s}-a_{s} \alpha}{b_{s}},
$$

$d_{s}, a_{s}, b_{s}$ being positive numbers, known from the choice of $s$.

We choose $\beta_{0}=\beta /\left(12 C_{s}\right)$, decreasing the value of $\beta$ if necessary to get $\beta_{0}<d_{s} / a_{s}$. Using the hypothesis on $K$ :

$$
\lim _{y \rightarrow \infty} y \int_{y}^{\infty} K(u)(2 u)^{(s-3) / 2} d u=0
$$

that is, $\forall \varepsilon>0, \exists M_{0}, \forall M>M_{0}$,

$$
M \int_{M}^{\infty} K(u)(2 u)^{(s-3) / 2} d u<\varepsilon \int_{0}^{\infty} K(u)(2 u)^{(s-3) / 2} d u,
$$

we choose $\varepsilon=\inf \left(\beta_{0} b_{s} /\left(4 d_{s}\right), 1 / 4\right)$; then $M_{0}$ is known.

Next, we choose a positive $M$ such that

$$
M>\max \left(M_{0}, a_{s} \beta_{0} / d_{s}, \beta_{0}, 1\right)
$$


and

Then $H$ is known and

$$
\alpha=\frac{\beta_{0}}{M} .
$$

$$
\frac{\alpha}{4 H}=\frac{\beta_{0} b_{s}}{4\left(d_{s} M-a_{s} \beta_{0}\right)}
$$

thus,

$$
\frac{\alpha}{4 H}>\frac{\beta_{0} b_{s}}{4 M d_{s}},
$$

and from the choices of $\varepsilon$ and $M$,

$$
\int_{M}^{\infty} K(u)(2 u)^{(s-3) / 2} d u<\frac{\alpha}{4 H} \int_{0}^{\infty} K(u)(2 u)^{(s-3) / 2} d u .
$$

We shall use this inequality at the end of the proof.

We choose the integer

$$
k_{n}=\left[\frac{h_{n}^{-1}}{\sqrt{2 M}\left(C_{s} 2^{s}\right)^{1 /(s-1)}}\right]
$$

and let

$$
\varrho_{n}=\frac{k_{n}^{-1}}{\left(C_{s} 2^{s}\right)^{1 /(s-1)}} .
$$

Then, for large $n, 2^{s} k_{n}^{s-1}>1 /\left(3 M C_{s}\right)$.

For large $n$ in $N_{1}$, we have $k_{n}^{s-1}>\beta^{\prime} n / \log n$, where $\beta^{\prime}=\beta /\left(3 M C_{s}\right)=$ $4 \alpha$. This inequality is valid if $\beta$ is chosen small enough.

We make a partition of $C$, similar to the partition defining $\widehat{f}_{n}$ on $S$ : without going into details, we simply note that we divide $[0, \pi / 2]$ for $\theta_{1}$ and the partition is associated with the integer $2 k_{n}$.

Let $K_{n}$ be the number of elements in this partition; $K_{n}$ is equivalent to $2^{s} k_{n}^{s-1}$. For each element, the area is equivalent to $C_{s} \varrho_{n}^{s-1}$.

We obtain a similar result to Proposition 1 of [3]:

Let $J_{n}$ be the exact number of $\Delta_{n, t}, t=1, \ldots, K_{n}$, containing no element of the sample. Then for every $\varepsilon>0$,

$$
\lim _{n \rightarrow \infty} P\left[1 \leq J_{n} \leq \varepsilon K_{n}\right]=1 .
$$

We can also state (cf. [3]):

Let $j$ an integer in $\left\{1, \ldots, K_{n}\right\}$ and integers $t_{1}, \ldots, t_{j}$ be such that

$$
1 \leq t_{1}<\ldots<t_{j} \leq K_{n}
$$

Let $V_{n}\left(t_{1}, \ldots, t_{j}\right)$ be the event: each $\Delta_{n, t}, t=t_{1}, \ldots, t_{j}$, is empty, while each among the others contains at least a point of the sample; the hypothesis $h_{n}^{1-s}=o(n)$ implies $K_{n}=o(n)$. Let $\alpha^{\prime}$ and $\alpha^{\prime \prime}$ be the positive numbers defined in [3]; suppose $n$ is so large that $K_{n}<\alpha^{\prime} n$, and let $\nu$ be an integer 
such that $\left[\alpha^{\prime} n\right]+1 \leq \nu \leq \alpha^{\prime \prime} n$; let $\nu_{n}$ be the number of $X_{i}$ 's belonging to $C$. Then the distribution of each $X_{i}(i=1, \ldots, n)$ conditioned by the event

$$
\mathcal{E}_{n}\left(\nu ; t_{1}, \ldots, t_{j}\right)=\left\{\nu_{n}=\nu\right\} \cap V_{n}\left(t_{1}, \ldots, t_{j}\right)
$$

admits the density

$$
f^{*}(x)= \begin{cases}\frac{n-\nu}{n} \frac{f(x)}{1-\alpha K_{n} C_{s} \varrho_{n}^{s-1}} & \text { if } x \in S-C, \\ \frac{\nu}{n \alpha} \frac{f(x)}{\left(K_{n}-j\right) \varrho_{n}^{s-1} C_{s}} & \text { if } x \in C-\bigcup_{r=1}^{j} \Delta_{n, t_{r}}, \\ 0 & \text { if } x \in \bigcup_{r=1}^{j} \Delta_{n, t_{r} .} .\end{cases}
$$

We now conclude as in [3]. Let

$$
\psi(x)=E\left[\widetilde{f}_{n}(x) \mid \mathcal{E}_{n}\left(\nu ; t_{1}, \ldots, t_{j}\right)\right] .
$$

Then

$$
\begin{aligned}
\psi(x)= & \frac{1}{h_{n}^{s-1} C_{K, s}\left(h_{n}\right)} \int_{S-C} K\left(\frac{1-(x, u)}{h_{n}^{2}}\right) \frac{n-\nu}{n(1-\alpha)} f(u) d \mu(u) \\
& +\frac{1}{h_{n}^{s-1} C_{K, s}\left(h_{n}\right)} \\
& \times \int_{C-\cup_{r=1}^{j} \Delta_{n, t_{r}}} K\left(\frac{1-\langle x, u\rangle}{h_{n}^{2}}\right) \frac{\nu}{n \alpha\left(K_{n}-j\right) \varrho_{n}^{s-1} C_{s}} f(u) d \mu(u) .
\end{aligned}
$$

Let $\varepsilon$ be in ]0,1[, and suppose $1 \leq j \leq \varepsilon K_{n}$. Then, for large $n$,

$$
\left(K_{n}-j\right) \varrho_{n}^{s-1} C_{s}>1-\varepsilon,
$$

and we can bound

$$
\begin{aligned}
\psi(x) \leq & \frac{1}{h_{n}^{s-1} C_{K, s}\left(h_{n}\right)} \int_{S-C} K\left(\frac{1-\langle x, u\rangle}{h_{n}^{2}}\right) \frac{1-\alpha^{\prime}}{1-\alpha} f(u) d \mu(u) \\
& +\frac{1}{h_{n}^{s-1} C_{K, s}\left(h_{n}\right)} \int_{C-\cup_{r=1}^{j} \Delta_{n, t_{r}}} K\left(\frac{1-\langle x, u\rangle}{h_{n}^{2}}\right) \frac{\alpha^{\prime \prime}}{\alpha(1-\varepsilon)} f(u) d \mu(u) .
\end{aligned}
$$

If $\alpha^{\prime}$ and $\alpha^{\prime \prime}$ are chosen such that

$$
\frac{1-\alpha^{\prime}}{1-\alpha}<1+2 \varepsilon \quad \text { and } \quad \frac{\alpha^{\prime \prime}}{\alpha(1-\varepsilon)}<1+2 \varepsilon
$$

then

$$
\psi(x) \leq \int_{S-\cup_{r=1}^{j} \Delta_{n, t_{r}}} \frac{1+2 \varepsilon}{h_{n}^{s-1} C_{K, s}\left(h_{n}\right)} K\left(\frac{1-\langle x, u\rangle}{h_{n}^{2}}\right) f(u) d \mu(u) .
$$


Let us choose $x=\xi$, corresponding to $\theta_{1}=0$, a pole of $\Delta_{n, t_{1}}=\Delta_{k_{n}, 0}$. We obtain

$$
\psi(\xi) \leq \frac{1+2 \varepsilon}{h_{n}^{s-1} C_{K, s}\left(h_{n}\right)} \int_{S-\cup_{r=1}^{j} \Delta_{n, t_{r}}} K\left(\frac{1-\langle\xi, u\rangle}{h_{n}^{2}}\right) f(u) d \mu(u),
$$

that is,

$$
\psi(\xi) \leq \frac{1+2 \varepsilon}{h_{n}^{s-1} C_{K, s}\left(h_{n}\right)} \int_{S-\cup_{r=1}^{j} \Delta_{n, t_{r}}} K\left(\frac{1-\cos \theta_{1}}{h_{n}^{2}}\right) f\left(\theta_{1}, \ldots, \theta_{s-1}\right) d \mu(\theta) .
$$

Let $D^{\prime \prime}$ be the image of the integration domain under the change of variable $u=\left(1-\cos \theta_{1}\right) / h_{n}^{2}$. Then

$$
\psi(\xi) \leq \frac{1+2 \varepsilon}{C_{K, s}\left(h_{n}\right)} \frac{2 \pi^{(s-1) / 2}}{\Gamma((s-1) / 2)}(\sup f) \int_{D^{\prime \prime}} K(u)(2 u)^{(s-3) / 2} d u .
$$

The image of the cap $\Delta_{n, t_{1}}$ has no common point with $D^{\prime \prime}$ and is the interval $\left[0, \varrho_{n}^{2} /\left(2 h_{n}^{2}\right)\right]$. Thus

$$
\psi(\xi) \leq(1+2 \varepsilon)(\sup f) \frac{2 \pi^{(s-1) / 2}}{\Gamma((s-1) / 2) C_{K, s}\left(h_{n}\right)} \int_{\varrho_{n}^{2} /\left(2 h_{n}^{2}\right)}^{\infty} K(u)(2 u)^{(s-3) / 2} d u .
$$

Remembering that

$$
\frac{\varrho_{n}^{2}}{2 h_{n}^{2}}=\frac{1}{\left(C_{s} 2^{s}\right)^{2 /(s-1)} 2 h_{n}^{2} k_{n}^{2}} \quad \text { and } \quad k_{n}^{2} \leq \frac{1}{2 M h_{n}^{2}\left(C_{s} 2^{s}\right)^{2 /(s-1)}}
$$

we have $\varrho_{n}^{2} / 2 h_{n}^{2} \geq M$ and

$$
\psi(\xi) \leq(1+2 \varepsilon)(\sup f) \frac{2 \pi^{(s-1) / 2}}{\Gamma((s-1) / 2) C_{K, s}\left(h_{n}\right)} \int_{M}^{\infty} K(u)(2 u)^{(s-3) / 2} d u .
$$

Recall also that

$$
\int_{M}^{\infty} K(u)(2 u)^{(s-3) / 2} d u<\inf \left(\frac{\alpha}{4 H}, \frac{\alpha}{4 M}\right) \int_{0}^{\infty} K(u)(2 u)^{(s-3) / 2} d u
$$

and, from the definition of $M$,

$$
\int_{M}^{\infty} K(u)(2 u)^{(s-3) / 2} d u<\inf \left(\frac{\alpha}{4 H}, \frac{1}{4}\right) \int_{0}^{\infty} K(u)(2 u)^{(s-3) / 2} d u .
$$

But $\sup f=\sup (\alpha, H)$ and thus

$$
\psi(\xi)<\left(\frac{1}{2}+\varepsilon\right) \frac{\left[2 \pi^{(s-1) / 2} / \Gamma((s-1) / 2)\right] \int_{0}^{\infty} K(u)(2 u)^{(s-3) / 2} d u}{C_{K, s}\left(h_{n}\right)} \alpha
$$


and for large $n$,

$$
\psi(\xi)<\left(\frac{1}{2}+\varepsilon\right) \frac{\alpha}{1-\varepsilon^{\prime}} .
$$

Choosing $\varepsilon=\varepsilon^{\prime}=1 / 10$, for large $n$, we get $\psi(\xi)<\frac{2}{3} \alpha$, and the end of the proof is similar to [3].

2. Sufficient condition for convergence. In this part, too, we proceed as in $[3]$.

We recall that a function defined on $\mathbb{R}^{+}$is called $\pi_{m}$-simple if, for a fixed integer $m$, it is constant on each element of the partition $\pi_{m}$, where

$$
\pi_{m}=\left\{I_{m, j}=\left[j / 2^{m},(j+1) / 2^{m}[: j \in \mathbb{N}\} .\right.\right.
$$

We suppose that $K$ is chosen such that there exist two sequences $\varphi_{m}^{+}$and $\varphi_{m}^{-}$of $\mathbb{R}^{+}$-integrable $\pi_{m}$-simple functions with

$$
\varphi_{m}^{-} \leq \varphi_{m+1}^{-} \leq K \leq \varphi_{m+1}^{+} \leq \varphi_{m}^{+} \quad \text { for large } m .
$$

For instance, every function $K$ of bounded variation in the neighboorhood of infinity satisfies this condition.

We suppose, moreover, that $u^{(s-1) / 2} K(u)$ is decreasing for large $u$, and that $\int_{0}^{\infty} u^{(s-1) / 2} K(u) d u$ exists, with

$$
\begin{aligned}
\lim _{m \rightarrow \infty} \int_{0}^{\infty} u^{(s-3) / 2} \varphi_{m}^{+}(u) d u & =\lim _{m \rightarrow \infty} \int_{0}^{\infty} u^{(s-3) / 2} \varphi_{m}^{-}(u) d u \\
& =\int_{0}^{\infty} u^{(s-3) / 2} K(u) d u
\end{aligned}
$$

We are going to prove the following theorem:

If $K$ satisfies the above hypotheses and if $h_{n}^{1-s}=o(n / \log n)$, then for each element $f$ of $\mathcal{D}, \tilde{f}_{n}$ converges to $f$ uniformly almost completely.

We set

$$
\varphi_{m}^{+}=\sum_{j=0}^{\infty} \alpha_{m_{j}} \mathbf{1}_{I_{m_{j}}}, \quad \varphi_{m}^{-}=\sum_{j=0}^{\infty} \alpha_{m_{j}}^{\prime} \mathbf{1}_{I_{m_{j}}} .
$$

We can write

$$
\begin{aligned}
& \frac{1}{n h_{n}^{s-1} C_{K, s}\left(h_{n}\right)} \sum_{i=1}^{n} \sum_{j=0}^{\infty} \alpha_{m_{j}}^{\prime} \mathbf{1}_{I_{m_{j}}}\left(\frac{1-\left\langle x, X_{i}\right\rangle}{h_{n}^{2}}\right) \\
& \leq \widetilde{f}_{n}(x) \leq \frac{1}{n h_{n}^{s-1} C_{K, s}\left(h_{n}\right)} \sum_{i=1}^{n} \sum_{j=0}^{\infty} \alpha_{m_{j}} \mathbf{1}_{I_{m_{j}}}\left(\frac{1-\left\langle x, X_{i}\right\rangle}{h_{n}^{2}}\right) .
\end{aligned}
$$


Consider the event

$$
\left\{\mathbf{1}_{I_{m_{j}}}\left(\frac{1-\left\langle x, X_{i}\right\rangle}{h_{n}^{2}}\right)=1\right\}
$$

that is,

$$
\left\{\frac{j}{2^{m}} \leq \frac{1-\left\langle x, X_{i}\right\rangle}{h_{n}^{2}}<\frac{j+1}{2^{m}}\right\}
$$

or

$$
\left\{X_{i} \in B_{n, m, j+1, x}-B_{n, m, j, x}=C_{n, m, j, x}\right\},
$$

where $B_{n, m, j, x}$ (resp. $B_{n, m, j+1, x}$ ) is the spherical cap with pole $x$ and radius $a_{n}=\left(j / 2^{m-1}\right)^{1 / 2} h_{n}\left(\right.$ resp. $\left.b_{n}=\left((j+1) / 2^{m-1}\right)^{1 / 2} h_{n}\right)$. Let

$$
\tilde{f}_{n, m, j}(x)=\frac{\nu_{n, m, j, x}}{n C_{s} a_{n}^{s-1}} \quad \text { and } \quad \tilde{f}_{n, m, j+1}(x)=\frac{\nu_{n, m, j+1, x}}{n C_{s} b_{n}^{s-1}}
$$

be the spherical cap estimators corresponding to these two caps. When $j$ and $m$ are chosen, the hypothesis about $h_{n}$ implies the uniform almost complete convergence of these two estimators. For the chosen $j$ and $m$,

$$
\begin{aligned}
& \frac{\left(2^{m-1}\right)^{(s-1) / 2}}{n h_{n}^{s-1} C_{K, s}\left(h_{n}\right)} \sum_{i=1}^{m} \mathbf{1}_{I_{m_{j}}}\left(\frac{1-\left\langle x, X_{i}\right\rangle}{h_{n}^{2}}\right) \\
& =\frac{\left(2^{m-1}\right)^{(s-1) / 2}}{n h_{n}^{s-1} C_{K, s}\left(h_{n}\right)}\left(\nu_{n, m, j+1, x}-\nu_{n, m, j, x}\right) \\
& =\frac{C_{s}}{C_{K, s}\left(h_{n}\right)}\left[(j+1)^{(s-1) / 2} \widetilde{f}_{n, m, j+1}(x)-j^{(s-1) / 2} \widetilde{f}_{n, m, j}(x)\right] .
\end{aligned}
$$

So the preceding bounds allow us to write

$$
\begin{aligned}
\frac{C_{s}}{C_{K, s}\left(h_{n}\right)} \sum_{j=0}^{\infty} \alpha_{m j}^{\prime} & \frac{1}{\left(2^{m-1}\right)^{(s-1) / 2}} \\
& \times\left[(j+1)^{(s-1) / 2} \widetilde{f}_{n, m, j+1}(x)-j^{(s-1) / 2} \widetilde{f}_{n, m, j}(x)\right]-f(x) \\
\leq & \widetilde{f}_{n}(x)-f(x) \\
\leq & \frac{C_{s}}{C_{K, s}\left(h_{n}\right)} \sum_{j=0}^{\infty} \alpha_{m j} \frac{1}{\left(2^{m-1}\right)^{(s-1) / 2}} \\
& \times\left[(j+1)^{(s-1) / 2} \widetilde{f}_{n, m, j+1}(x)-j^{(s-1) / 2} \widetilde{f}_{n, m, j}(x)\right]-f(x) .
\end{aligned}
$$


Consider, first, the upper bound of $\widetilde{f}_{n}(x)-f(x)$. We can write it as

$$
\begin{aligned}
\frac{C_{s}}{C_{K, s}\left(h_{n}\right)} & \sum_{j=0}^{\infty} \frac{\alpha_{m, j}}{2^{(m-1)(s-1) / 2}} \\
\times & \left\{(j+1)^{(s-1) / 2}\left[\widetilde{f}_{n, m, j+1}(x)-f(x)\right]-j^{(s-1) / 2}\left[f_{n, m, j}(x)-f(x)\right]\right\} \\
+ & f(x)\left\{\frac{C_{s}}{C_{K, s}\left(h_{n}\right)} \sum_{j=0}^{\infty} \frac{\alpha_{m, j}}{2^{(m-1)(s-1) / 2}}\left[(j+1)^{(s-1) / 2}-j^{(s-1) / 2}\right]-1\right\} .
\end{aligned}
$$

Recall that

$$
\begin{aligned}
\lim _{n \rightarrow \infty} C_{K, s}\left(h_{n}\right) & =\frac{2 \pi^{(s-1) / 2}}{\Gamma((s-1) / 2)} \int_{0}^{\infty}(2 u)^{(s-3) / 2} K(u) d u, \\
C_{s} & =\frac{2 \pi^{(s-1) / 2}}{(s-1) \Gamma((s-1) / 2)} .
\end{aligned}
$$

Moreover,

$$
(s-1) \int_{0}^{\infty} \varphi_{m}^{+}(u)(2 u)^{(s-3) / 2} d u=\sum_{j=0}^{\infty} \frac{\alpha_{m j}}{2^{(m-1)(s-1) / 2}}\left[(j+1)^{(s-1) / 2}-j^{(s-1) / 2}\right] .
$$

From the hypotheses about $K$, there exists an integer $m_{0}$ such that, for $m>m_{0}$,

$$
\int_{0}^{\infty} \varphi_{m}^{+}(u)(2 u)^{(s-3) / 2} d u<(1+\varepsilon) \int_{0}^{\infty} K(u)(2 u)^{(s-3) / 2} d u .
$$

Thus, for $n>n_{0}$ and $m>m_{0}$, the coefficient of $f(x)$ is smaller than an arbitrary positive number $\eta$.

Let us choose $m>m_{0}$. The hypotheses about $u^{(s-1) / 2} K(u)$ imply that, for each $\varepsilon>0$, there exists a finite subset $J$ of $\mathbb{N}$ such that

$$
\sum_{j \notin J} \alpha_{m j}\left[j^{(s-1) / 2}+(j+1)^{(s-1) / 2}\right]<\varepsilon .
$$

Let $H$ be an upper bound for $f$. For $n>n_{0}, \widetilde{f}_{n}(x)-f(x)$ is smaller than

$$
\begin{aligned}
& \frac{C_{s}}{C_{K, s}\left(h_{n}\right)} \sum_{j \in J} \frac{\alpha_{m j}}{2^{(m-1)(s-1) / 2}}(j+1)^{(s-1) / 2}\left|\widetilde{f}_{n, m, j+1}(x)-f(x)\right| \\
& +\frac{C_{s}}{C_{K, s}\left(h_{n}\right)} \sum_{j \in J} \frac{\alpha_{m j}}{2^{(m-1)(s-1) / 2}} j^{(s-1) / 2}\left|\widetilde{f}_{n, m, j}(x)-f(x)\right|+2 H \varepsilon \frac{C_{s}}{C_{K, s}\left(h_{n}\right)}+H .
\end{aligned}
$$

The end of proof is similar to [3].

The lower bound for $\widetilde{f}_{n}(x)-f(x)$ is obtained analogously. 


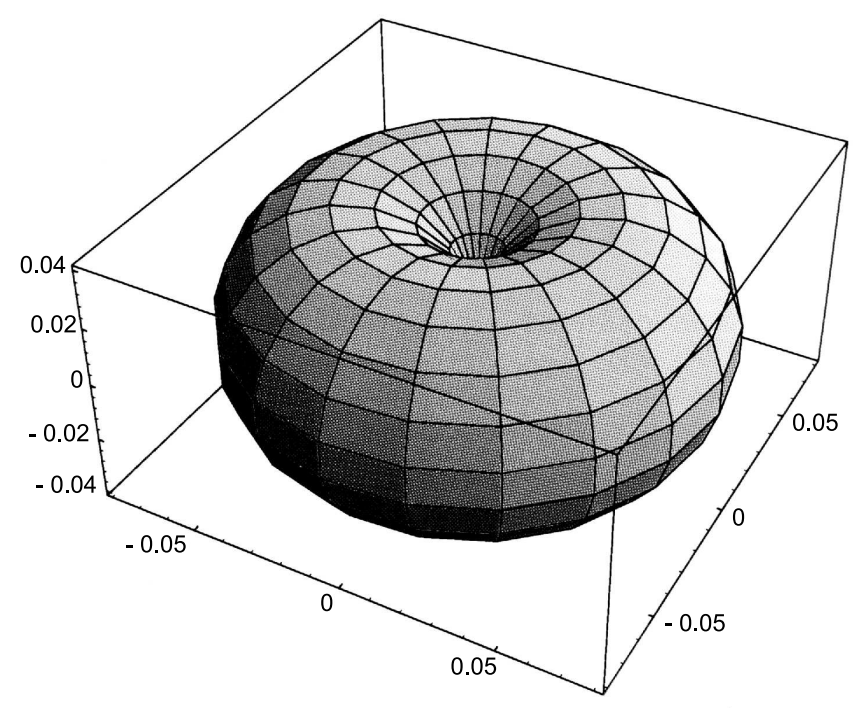

$$
f\left(\theta_{1}, \theta_{2}\right)=\frac{1}{\pi^{2}} \sin \theta_{1}
$$

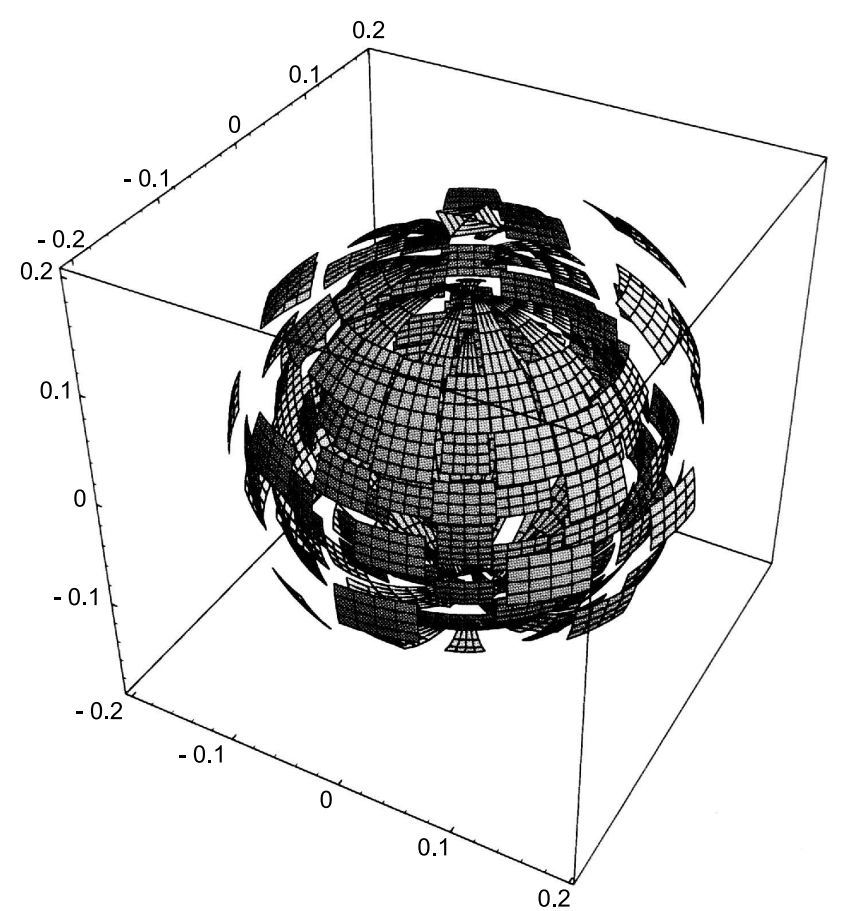

The histogram estimator 


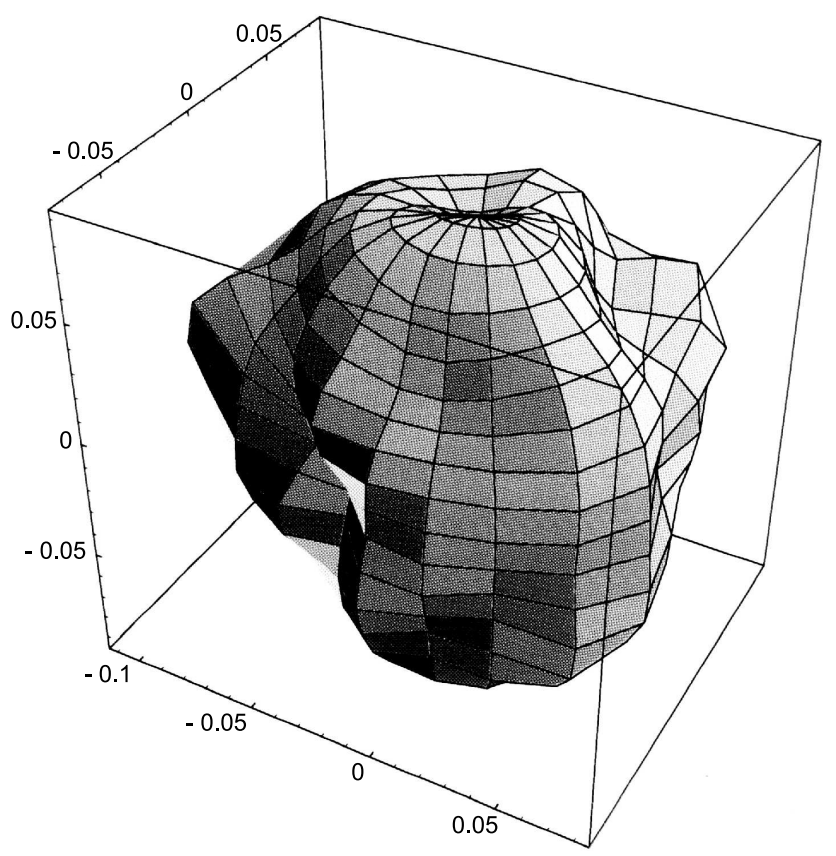

The kernel estimator

D. Simulation results. We now study the performance of these estimators by simulation methods, for the density

$$
f\left(\theta_{1}, \theta_{2}\right)=\frac{1}{\pi^{2}} \sin \theta_{1}
$$

with $s=3$.

The histogram estimate is calculated from a sample of size $n=5000$, with $k=\sqrt{n} / \log n$.

The kernel estimate is calculated from a sample of size $n=1000$, with $K(u)=\frac{1}{2} e^{-u}(u \geq 0)$ and $h_{n}=(\log n) / \sqrt{n}$.

\section{References}

[1] Z. D. Bai, C. Radakrishna Rao and L. C. Zhao, Kernel estimators of density function of directional data, J. Multivariate Anal. 27 (1988), 24-39.

[2] M. Bertrand-Retali, Convergence uniforme stochastique d'un estimateur d'une densité de probabilité dans $\mathbb{R}^{s}$, C. R. Acad. Sci. Paris Sér. A 278 (1974), 451-453.

[3] -, Convergence uniforme d'un estimateur de la densité par la méthode du noyau, Rev. Roumaine Math. Pures Appl. 23 (1978), 361-385.

[4] J. Geffroy, Sur l'estimation d'une densité dans un espace métrique, C. R. Acad. Sci. Paris Sér. A 278 (1974), 1449-1452.

[5] P. Hall, G. S. Watson and J. Cabrera, Kernel density estimation with spherical data, Biometrika 74 (1987), 751-762. 
[6] F. H. Ruymgaart, Strong uniform convergence of density estimators on spheres, J. Statist. Plann. Inference 23 (1989), 45-52.

[7] G. S. Watson, Statistics on Spheres, University of Arkansas Lecture Notes in Math. Sci., Wiley, 1983.

MONIQUE BERTRAND-RETALI

LARBI AIT-HENNANI

UNIVERSITÉ DE ROUEN

UFR DES SCIENCES MATHÉMATIQUES

ANALYSE ET MODĖLES STOCHASTIQUES

URA CNRS 1378

76821 MONT SAINT AIGNAN CEDEX, FRANCE 